\title{
Die Herrschaft des politischen Islam als eine Form des Totalitarismus »Die Islamische Republik Iran«
}

Die Wahl des neuen iranischen Präsidenten Khatami wurde hierzulande als Anzeichen einer »Öffnung « wahrgenommen, die eine »Liberalisierung « des Regimes und die Herausbildung einer »islamischen Zivilgesellschaft « erwarten lasse. Die Schlüsselfrage, ob sich die strukturellen Grundlagen des vermeintlichen »Gottesstaates « nach dem Tode Khomeinis verändert haben, wurde jedoch nicht diskutiert. Zwar wurde die Herrschaft der schiitischen Geistlichkeit schon verschiedentlich als »totalitär« bezeichnet, der Frage, inwieweit eine solche Charakterisierung berechtigt ist, wurde bisher jedoch nicht nachgegangen. In diesem Beitrag werden zunächst die Entwicklung und die strukturellen Grundlagen der Mullah-Herrschaft im Iran untersucht. Daran anschließend wird gezeigt, daß diese Herrschaftsform, obwohl sie weder als »faschistisch « noch als »kommunistisch « gelten kann, trotzdem eine Reihe von Kriterien erfüllt, die als charakteristisch für eine totalitäre Diktatur gelten. Solange das Prinzip der absoluten Herrschaft der Geistlichkeit in der iranischen Verfassung festgelegt ist und das iranische Strafgesetz in der khomeinistischen Interpretationen des Islam zur Geltung kommt, ist jede Rede über eine islamische Zivilgesellschaft eine demagogische Farce. Werden zur Unterstützung einer angeblichen Liberalisierung die Wirtschaftsbeziehungen ausgebaut, so läuft dies darauf hinaus, Macht der herrschenden Mullahs aller Fraktionen zu stabilisieren (vgl. auch Heinz 1997).

\section{Die Revolution im Iran}

Die Revolution von 1978/79 hat gezeigt, daß die traditionelle Gesellschaft sich nicht mit Gewalt in das 20. Jahrhundert transformieren ließ; die von der »sultanistischen« Schahdiktatur (Katouzian 1998: 185) angestrebte Modernisierung war gescheitert. Die städtische Montageindustrie hatte keinen inneren Markt geschaffen. Die Kapitalisierung der Landwirtschaft hatte zur 
Bereicherung von wenigen Großgrundbesitzern geführt. Die Landflucht in die Slums der Großstädte war das Merkmal eines nicht erfolgreich durchgesetzten Entwicklungsmodells. Das vom Militär und einem brutalen Geheimdienst, dem SAVAK (Zonis 1983: 601), getragene Modernisierungsprojekt exkludierte die Massen und provozierte die traditionalistisch orientierten Teile der Mittelschichten und ihre Intellektuellen. Die Diktatur des Schah-Regimes hatte es nicht erreicht, die westlichen Importe in die Lebensweise der Bevölkerung zu integrieren (Fukuyama 1992: 323). Westliche Werte wurden besonders von der Geistlichkeit als eine Bedrohung ihrer traditionellen Machtposition gesehen.

Seit 1976 befand sich die iranische Wirtschaft in einer schweren Rezession. Amerikanische Firmen pumpten Petrodollars aus dem Iran und dieser kaufte nutzlose Waffen, industrielle Güter und Konsumgüter für die neuen Mittelschichten ein (Rouleau 1980: 4). Die Intellektuellen des Schiismus griffen in die Mottenkiste der religiösen Tradition und verfochten das Ziel, der alten Sozialordnung der Kalifen wieder Geltung zu verschaffen. Durch die Kollision mit der technologisch überlegenen Gesellschaft des Westens entstand der Versuch der Rückbesinnung auf die »eigene Kultur«.

Der Schah hatte im Jahre 1974 die kalendarische Zeitrechnung in Erinnerung an die iranisch-archetypische Tradition der Dynastien auf das Jahr 2500 (Bernard/Khalilzad 1979: 235) nach vorne gedreht. Fünf Jahre später drehten die Islamisten die Uhr wieder zurück. Der Iran zählte das Jahr 1357 nach mohammedanischer Zeitrechnung. Wenn das mittelalterliche Europa in den »Bereichen der Kultur, Mathematik, Technik oder der Navigation « einen Teil seiner Kultur historisch gesehen vom Islam »geliehen « (Kennedy 1989: 30) hat, muß im Übergang zum 21. Jahrhundert davon ausgegangen werden, daß der politische Islam des Khomeinismus auf Verfallserscheinungen einer Weltreligion hinweist. In der sunnitischen Tradition errichtete Khomeini einen Gottesstaat (Gellner 1987: 287), der die diktatorischen Dimensionen des Schah-Regimes noch weit übertrifft. Ein islamischer Gottesstaat und ein reiner islamischer Mensch sollte den Iran vor der westlichen Dekadenz retten. Auch wenn der neue Präsident Khatami die Barbarei des politischen Islam als eine neue Zivilisation zu verkaufen versucht (Wahdathagh 1998b, 1999b), kann davon ausgegangen werden, daß ein Andalusien, das historisch für die tolerante Tradition des Islam steht, im khomeinistischen Iran kaum vorstellbar ist. Der politische Islam ist ein Phänomen und ein Teil der Moderne: Der Iran errichtet Atomkraftwerke, baut Mittelstreckenraketen mit einer Reichweite von $1300 \mathrm{~km}$ (IRNA 7.2.1998) und das iranische Nuklearprogramm soll bis 2003 in der Lage sein, eigene Atombomben zu bauen (Hashim 1995: 60).

Der Streik der iranischen Ölarbeiter war der Anfang vom Ende der »sultanistischen Diktatur« der Pahlavi-Dynastie. Fabriken und Ländereien wur- 
den besetzt. Studenten, Professoren, Schüler und Lehrer diskutierten über Befreiungsbewegungen in der Dritten Welt. Banken wurden überfallen, Kasernen gestürmt. Politische Organisationen rüsteten sich auf, um gegen eine der stärksten Armeen des Mittleren Ostens zu kämpfen. Die Massen stellten sich Panzern entgegen. Auch die US-Generalität konnte den Schah nicht mehr an der Macht halten (Huyser 1986). Nachdem die Armee einige hundert Demonstranten niedergeschossen hatte, kapitulierte der gesamte westlich ausgebildete Militärapparat, weil kein Befehl von oben kam.

Im »Frühling der Freiheit« organisierten sich säkulare Frauen in Frauenorganisationen und veranstalteten Seminare und Demonstrationen für die Gleichberechtigung der Geschlechter. Verschiedene liberal-demokratische Gruppen, wie der »Nationale Verband der Universitätsprofessoren«, der »Anwaltsverband « und der Schriftstellerverband hatten sich erneut formiert und wurden politisch aktiv. Unabhängige Zeitungen und Verlage aller politischer Schattierungen wurden gegründet. Die Paniranistische Partei und die sozialdemokratisch orientierten Organisationen hofften auf die langfristige Mobilisierung ihrer Anhängerschaft.

Kommunistische Organisationen träumten von der Zerschlagung des Weltkapitalismus, vertrauten aber Khomeini. Die marxistisch-leninistische Organisation der Volksfedajin schrieb einen offenen Brief an Khomeini und bat ihn auf die sozialen Belange der Arbeiterklasse zu achten, mäßigend auf die paramilitärischen islamistischen Gruppen einzuwirken, und sie aufzufordern, die kommunistischen Buchläden und Büros nicht mehr anzugreifen (Etellaat 21.1.1979: 2). Ansonsten schickten die kommunistischen Organisationen fleißig Kader in die Fabriken und auf das Land, um die Arbeiter und die Bauern für die sozialistische Weltrevolution zu gewinnen und spalteten sich in immer weitere Splittergruppen. Die bewaffnete Organisation der Volksmudjahedin kämpfte für einen »Sozialismus mit islamischem Antlitz«.

Viele Iraner träumten von einer Revolution, die die alten sozialen Strukturen der orientalischen Despotie auflösen und eine neue sozial gerechtere und demokratischere Gesellschaft schaffen würde. Die demokratische Revolution von 1979 ist jedoch im Keime erstickt und islamisiert worden.

Rund zehn Jahre nach der Revolution stellte Bernard Lewis die zweischneidige These auf, daß die islamische Revolution sich durchaus mit der französischen oder der russischen Revolution vergleichen lasse, weil eine Massenbewegung Verschiebungen im Gefüge der Macht bewirkt und einen Prozeß umfassenden Wandels eingeleitet habe (Lewis 1988: 154). Entwicklungen seien beschleunigt worden, die schon lange im Gange waren. Auch wenn die grausamen Morde aller Revolutionen sich gleichen mögen, unterscheiden sich Revolutionen in ihrem Ergebnis darin, ob eine demokratische Ordnung herbeigeführt wird oder ob der Gang der Geschichte wie in 
Rußland im Stalinismus oder wie im Fall des Iran im Khomeinismus mündet. Für Lewis schien es jedoch noch offen, ob der iranische Gottesstaat einen demokratischen Weg einschlagen würde. Tatsächlich war die iranische Revolution die erste, die eine Rückkehr zum 7. Jahrhundert nach Christus als erklärtes Ziel verfolgte. Im Vergleich zu anderen Revolutionen wirkte dies so grotesk, »als würde sich 1848 Papst Pius IX. zur Führung der römischen Revolution entschlossen haben« (Hobsbawm 1994: 565).

\section{Die Machtübernahme der Khomeinisten}

Der Politiker des säkularen Flügels »Nationalen Front « Schapur Bachtiar wurde am 31.12.1978 eingesetzt, um einen Kompromiß zwischen Khomeini und dem Schah-Regime herbeizuführen. Bachtiar verfocht als Tribun eines modernistischen Flügels der »Nationalen Front« einen Parlamentarismus plus dynastische Herrschaft. Die Lockerung des politischen Klimas kam jedoch viel zu spät. Bachtiar stornierte Waffenkäufe im Wert von 7 Milliarden Dollar, er stoppte den Verkauf von Öl an Israel und Südafrika und erklärte den Austritt des Iran aus CENTO. Verzweifelt nannte er Khomeini sogar den »iranischen Ghandi« (Abrahamian 1998: 485). Schapur Bachtiar mußte das Land am 9.2.1979, acht Tage nach der Ankunft Khomeinis, verlassen. Im August 1991 wurde er von khomeinistischen Todesschwadronen in Paris bestialisch ermordet.

Die Massen agierten zwar einheitlich gegen den Schah und riefen: »Der Schah muß weg «, sie blieben aber gespalten. Die Einheit forderte insbesondere Khomeini: »Hame ba ham - Alle zusammen«. Er forderte auch die Einheit des Wortes, die den Anfang der totalitären Diktatur des Khomeinismus schon anklingen ließ. Wenige Monate später wurde für alle Beteiligten sichtbar, daß Khomeini von den anderen forderte $i h m$ zu folgen. $\mathrm{Er}$ hatte gemeint: »Alle mit mir«.

Khomeini kam am 1. Februar 1979 mit dem Flugzeug aus dem französischen Exil in Teheran an und wurde von den Massen wie ein von Allah gesandter Retter, ein Prophet empfangen. Für manche gläubige Muslime war der zwölfte Imam vom Himmel herabgekommen. Sie hatten schon sein Gesicht im Vollmond gesehen. Fromm und fanatisch schrien die Massen: »Tod dem Schah«, »Khomeini unser Führer«. Kaum ein fortschrittlicher Intellektueller machte sich Gedanken, ob der schiitische Revolutionär, der die »Durchsetzung des ewigen, göttlichen Rechts « verfolgte, doch nicht den »Sunnismus unter dem Mantel des Schiismus« (Gellner 1987: 288) verkaufte, und welche diktatorischen Dimensionen eine sich mit göttlicher Wahrheit identifizierende Herrschaftsform für Individuum und Gesellschaft mit sich bringen konnte. Dies wird vor dem Hintergrund des Einheitsdenken Khomeinis verständlich, der den Widerspruch zwischen Sunniten und 
Schiiten mit einer panislamischen Politik des Welayat ${ }^{1}$ zu überwinden versprach (Buchta 1994: 566).

\section{Islamisierung}

Während die demokratischen und kommunistischen Organisationen noch von ihren jeweiligen Zielen träumten und sich gegenseitig befehdeten, bereitete der khomeinistische Teil innerhalb der »Kaste« der schiitischen Geistlichkeit bereits die totale Machtübernahme vor. Der Begriff »Kaste « wurde hier gewählt weil die Führungsriege keine spezifische Klasse vertritt. Die scheinbar gespaltene Führungsriege operiert in allen politischen Richtungen und verfügt über einen ideologischen Konsens: Die Erhaltung und Stärkung der Macht des Welayate Fagih - der Herrschaft der Rechtsgelehrten. Wie schnell die Herrschaft der Khomeinisten das Land erfaßte machen einige Ereignisse deutlich.

Am 16. Juli 1979 gab Khomeini den Befehl für den Einmarsch der Armee in die kurdische Stadt Marivan. Die Kurden kämpften für ihre kulturelle Autonomie innerhalb der iranischen Grenzen.

Am 7. August wurde die bürgerlich-liberale Zeitung »Ayandegan « (Nirumand/Daddjou 1987: 268) verboten. Die Islamisch-Republikanische Partei (IRP) begrüßte das Verbot von weiteren 22 Zeitungen und Zeitschriften (Shirazi 1992: 73). Die kommunistische Linke übte sich im antiimperialistischen Kampf und fürchtete zionistische Kräfte in den Redaktionsräumen der Zeitung; einen Aufruf der »Nationalen Front«, die vor der Zensur warnte und die Verteidigung der elementaren Bürgerrechte forderte, unterschrieb sie nicht.

Mit Zustimmung Khomeinis besetzte ein studentisches Revolutionskomitee am 4. November 1979 die US-amerikanische Botschaft, nahm etwa 70 Geiseln (Chubin 1982: 19) und forderte die Auslieferung des Schahs. In Massendemonstrationen wurden Tausende permanent mobilisiert und auf eine antiamerikanische Linie gebracht.

Die islamische Kulturrevolution begann schon mit den Säuberungen der Universitäten. Ein eigens installierter Revolutionsrat der Universität entschied über die Entlassungen von Professoren, Studenten und Personal. Schon am 29. Mai 1979 wurde eine Erklärung des Direktoriums veröffentlicht, in der es hieß, daß die Universität ein heiliger Ort sei und von Agenten des Kolonialismus gesäubert werden müßte (Etelaat 29. Mai 1979, S.9). Der Höhepunkt der »islamischen Kulturrevolution« war die SchlieBung der Teheraner Universität im April 1980.

1 Der politische Zwang zur totalen Unterwerfung unter mohammedanischer Herrschaft und die islamischen Kalifen und Sultane wird von Karl A. Wittfogel als »orientalische Despotie« bezeichnet. Rosa Luxemburg (1985) spricht von der »asiatischen Despotie«, wenn sie die »Wilajet« behandelt. 
Khomeini bezeichnete die Universitäten als »Zentren der Prostitution« und verbot jegliche politische Aktivität an den Hochschulen (Momeni 1998a: 214). Der in deutschen Medien als »Reformer « bekannt gewordene islamistische Staatstheoretiker Sorusch und der heutige Präsident Mohammad Khatami gehörten zu den Befürwortern dieser kulturrevolutionären Säuberungen. Als Khatami 1982 das Amt des Ministers für »Kultur und islamische Führung « (Kooroshy 1998: 10) bekleidete, setzte er die Politik der Islamisierung der Gesellschaft in allen Bereichen mit größter Härte durch. Die Konsequenz der Islamisierung war die totale Eliminierung der Andersdenkenden aus der öffentlichen Sphäre. Im Februar 1983 wurde die moskauorientierte Tudeh-Partei (Chubin 1983: 933) und ihre Jugendorganisation, die »Volksfedajin-Mehrheit«, als letzte oppositionelle Organisation verboten. Die Tudeh-Partei stützte sich auf die Positionen eines sowjetischen Entwicklungsstrategen (Olianowsky 1979), der Khomeini zwar nicht als einen Sozialisten, aber als einen Antiimperialisten, einen Feind des imperialistischen Feindes schätzte. Die khomeinistische Basis sei die revolutionäre Kleinbourgeoisie, propagierte die Tudeh-Partei. Tatsächlich besteht die soziale Basis der Kaste der herrschenden Geistlichkeit aus den rückschrittlichsten traditionellen Schichten der iranischen Gesellschaft, das Lumpenproletariat eingeschlossen. Mitte Mai 1983 wurden führende Parteimitglieder der Tudeh-Partei, wahrscheinlich unter Drogen, im staatlichen Fernsehsender vorgeführt. Sie bereuten einer »fremden Ideologie « (Abrahamian 1993:88) angehangen zu haben.

In den Massenhinrichtungen von 1980-82 und 1988 wurden Tausende verhaftet und in den Gefängnissen gefoltert und hingerichtet (Baradaran 1999; Parsipur 1996; Abrahamian 1997).

Der Krieg gegen den Irak war nach Khomeini ein »Geschenk Gottes«, obwohl er weitere Konflikte in der Region auszulösen drohte, wie Raketenangriffe gegen Israel, oder die Einmischung der damaligen Supermächte in den Grenzkonflikt (Wright 1980/81: 301). Der Friedensvertrag war für den »alten Mann« Khomeini wie »ein Becher Gift«, den er austrinken mußte, wie er selbst zugab (Momeni 1998a: 330). Khomeini wußte das Feindbild Amerika zu instrumentalisieren, um die Liquidierung der politischen Feinde im Inland zu legitimieren (Ebd.: 273). Die Iran/Contra-Affäre (Leaman 1988) zeigte, daß der antiimperialistische Kampf auch der BanisadrRegierung unter Khomeini seine Grenzen beim Waffenhandel hatte. Tatsächlich hatte Khomeini den alten Staatsapparat, die Armee, den Geheimdienst SAVAK (heute VEVAK) und das Bankwesen geerbt. Das Problem des Zinsverbotes der Banken wurde mit einem leichten Kniff der Erhebung von Gebühren gelöst. In allen staatlichen Einrichtungen bis hin zur Armee wurden Geistliche als Agenten des neuen Systems installiert, die die Islamisierung der alten Administration garantieren sollten. 


\section{Drei politische Säulen der Islamischen Republik Iran}

Parallel zur provisorischen (ersten) Regierung von Bazargan (Mahrad 1983: 62; Buchta 1995b: 588) konkurrierte der islamische Revolutionsrat um die Macht im Staat. Bazargan und die Bewegung der »Nehzate Azadi«, die mit seinem Namen im Zusammenhang gebracht wird, gehörte dem traditionalistischen Flügel der »Nationalen Front « an. Der Biograph von Rafsandjani, Massud Rasavi schreibt zu Recht, daß in der ersten Phase der Revolution neben Khomeini, der islamische Revolutionsrat und die provisorische Regierung als die drei Säulen der Islamischen Republik betrachtet werden können (Rasavi 1997: 221). Die Widersprüche innerhalb dieser drei Säulen des Mullah-Regimes existieren bis heute.

$\mathrm{Zu}$ den Aufgaben des islamischen Revolutionsrates gehörten u.a. der Entwurf einer Verfassung, die Bildung einer konstituierenden Versammlung und Säuberungen der Institutionen, der Ministerien, der gesamten staatlichen Bürokratie von »frevlerischen Elementen«. Obwohl aus dem Umfeld von Bazargan zunächst ein Verfassungsentwurf vorgelegt wurde, in dem die Geistlichkeit nicht die alleinige Macht im Staate übernehmen sollte, forderte der heute als Oppositioneller geltende Ayatollah Montazeri am 11.7.1979, daß der Staatspräsident ein schiitischer Mojtahed sein sollte. Ein solcher Großayatollah steht an der Spitze der schiitischen Geistlichkeit und wird zum »Vorbild « (Marjae taglide motlaq), das von allen Geistlichen und Muslimen nachgeahmt werden muß (Buchta 1995a: 450f). Auch der Hauptrivale Montazeris, der gegenwärtige religiöse Führer des Iran, Hojatoleslam Khamenei forderte im Konsens mit den beiden »Vorbildern « Khomeini und Montazeri den Eingang des Prinzips der Welayate Fagih, der Herrschaft der Rechtsgelehrten, in die Verfassung (Shirazi 1992: 35).

Bazargan versuchte die Krise zu meistern und setzte sich für die Vereinheitlichung der provisorischen Regierung mit dem islamischen Revolutionsrat ein. Infolge dessen wurde Rafsandjani Sekretär des Staatsministers. Der Schein-»Pluralismus « der Kaste der Islamisten existierte bereits im Revolutionsrat, wenn man sich die Liste der zunächst geheim gehaltenen Mitglieder genauer betrachtet. Damals gehörten noch u.a. Montazeri, Khamenei, Beheschti, Rafsandjani, Banisadr, Bahonar, Mahdavi-Kani, Habibi, Sahabi dazu (Rasavi 1997: 231). Einige aus diesem Kreis wurden später entmachtet oder ermordet.

Banisadr hatte das Konzept einer göttlichen Wirtschaftsordnung (Banisadr 1979) entworfen, zugeschnitten auf den khomeinistischen Gottesstaat. Dennoch wurde er am 21. Juni 1981 mit Zustimmung der anderen Mitglie- 
der und des Majlis des islamischen Rates ${ }^{2}$ abgesetzt. Banisadr war zunächst von Khomeini als Wirtschaftsminister in die Regierung Bazargan delegiert worden (Hiro 1987: 125). Nachdem er zum Präsidenten gewählt worden war, wollte er die Macht des islamischen Revolutionsrates einschränken. Khomeini sprach schließlich ein Machtwort: »In diesen Tagen mehren sich die Angriffe gegen die traditionelle Geistlichkeit. Die Beleidigung der Geistlichkeit bedeutet eine Schwächung der Unabhängigkeit, der Freiheit und des Islam.« Jeder, der so handle, gehe den Weg des gestürzten Schah (Rasavi 1997: 235). Diese Warnungen richteten sich gegen die Organisation der Volksmudjahedin und gegen Banisadr.

Der Revolutionsrat, der hauptsächlich aus Geistlichen bestand, konnte, indem er sich von Anbeginn auf paramilitärische islamistische Organisationen wie die Revolutionskomitees und Revolutionsgerichte stützte, Aufgaben der staatlichen Verwaltung wahrnehmen.

Ajatollah Beheschti, Mitglied des islamischen Revolutionsrates, wurde am 29.6.1981 durch ein Bombenattentat auf die Zentrale der IslamischRepublikanischen Partei, gemeinsam mit 71 anderen Parteimitgliedern getötet. Präsident Rajai, der Nachfolger von Banisadr und Hojatoleslam Mohammad Javad Bahonar, Generalsekretär der IRP starben am 30.8.1980 ebenfalls durch ein Bombenattentat. Die Frage ist bis heute offen, ob tatsächlich die oppositionellen und bewaffneten Volksmudjahedin, die später von Paris und dann von Bagdad aus gegen die Mullahs operierten, die Bomben gelegt hatten, oder ob die Attentate den Mudjahedin angehängt wurden, um auch sie zu eliminieren. Ajatollah Taleghani fungierte als eine Brücke zwischen dem Islamischen Revolutionsrat und zur Nehzate Azadi, die Partei Bazargans, dem Vertreter der rückschrittlich-traditionellen Bazari-Bourgeoisie. Taleghani scheute sich aber auch nicht die Sowjetunion gegenüber den USA zu verteidigen. Er wurde daher von der Tudeh-Partei als fortschrittlich eingestuft. Khomeinisten galten grundsätzlich als Feinde des imperialistischen Feindes, die USA, und wurden daher von den iranischen Kommunisten als objektive Koalitionspartner betrachtet.

Auch Ezzatollah Sahabi der heute als Vertreter der »legalen Opposition « gilt, arbeitete damals im islamischen Revolutionsrat. Dies soll verdeutlichen, daß die Vielfalt der khomeinistischen Kräfte vom ersten Jahr der Revolution existierte, was aber nicht bedeutet, daß er die Vielfalt der gesellschaftlichen Realität des Iran widergespiegelt hätte. Feststeht, daß der Revolutionsrat sogar den islamistischen Flügel der »Nationalen Front«, die längst atomisiert war, mit dem Sturz der provisorischen Regierung von Ba-

2 Diese Institution wird fälschlicherweise von einigen Iran-Experten als Parlament bezeichnet. Dies trifft nicht zu, weil die in den Majlis gewählten Kandidaten nicht vom Volk aufgestellt werden, sondern aufoktrojierte Kandidaten sind, die gewählt werden können. 
zargan entmachtet hatte. Bazargan konnte die Besetzung der US-Botschaft nicht gutheißen und trat zwei Tage danach am 6. November 1979 zurück. Khomeini forderte die »Einheit des Direktoriums « und versprach der Bevölkerung materiellen Wohlstand für alle Iraner (Nasri 1983: 629).

Trotz all dieser Widersprüche haben diese Kräfte gemeinsam die Potentiale einer demokratischen Zivilgesellschaft beseitigt, so wie es Khomeini gefordert hatte. Am 12. Juli 1980 erklärte Beheschti die Arbeit des islamischen Revolutionsrates für beendet. Zwei neue Institutionen, der Wächterrat und der Majlis wurden ins Leben gerufen. Aus diesen internen Säuberungen trat Rafsandjani als Sieger hervor.

\section{Der Sieg der Islamisch-Republikanischen-Partei (IRP)}

Die Geistlichkeit sicherte ihre Machtposition Schritt für Schritt ab. Am 30./31 März 1979 dürfte das Volk Nein zum Schahregime und Ja zur Islamischen Republik sagen. Am 3.8.1979 führten die khomeinistischen Wahlen zur »verfassunggebenden Versammlung «, d.h. zum Sieg der schiitischen Geistlichkeit. Dieser Triumph wurde am 2. Dezember 1979 durch ein weiteres Referendum, das die neue Verfassung, die umfassende Vollmachten für Khomeini und die klerikale Kaste garantierte, abgesichert.

Schon am 4. Februar hatte die khomeinistische Gruppe der »kämpfenden Geistlichkeit« in einer Erklärung bekannt gegeben: »Der Islam ist nicht nur eine Religion, sondern ein soziales und politisches System « (Etelaat 4.2.1979: 8). Diese Worte wurden nicht ernst genommen. Allerdings verhinderten Khomeini und seine Anhänger genau in diesem Sinne die Bildung einer »konstituierenden Versammlung «. Diese hätte eine Wahl der von der Bevölkerung aufgestellten und gewählten Kandidaten ermöglicht. Nach und nach wurden alle vom khomeinistischen System unabhängigen gesellschaftspolitischen Instanzen verfolgt und verboten. Lediglich die Islamisch-Republikanische Partei (IRP) blieb. Sie fungierte als eine politische Dachorganisation vieler islamischen Gemeinschaften im gesamten Land (Sciolioni 1983: 897).

Der islamistische Historiker Rasawi schreibt, daß sechs Personen des Zentralkomitees vor der Gründung der Partei von Khomeini ernannt wurden, um in den Revolutionsrat einzutreten. Als Bazargan noch mitregierte, habe der Revolutionsrat die Rolle einer verfassungsgebenden Versammlung inne gehabt. Nach seinem Rücktritt verpflichteten sich die von Khomeini auserwählten Personen zusätzlich zu konkreten Arbeiten in den revolutionären Organen, wie bei den Pasdaran, den Revolutionsgerichten etc. Auch Banisadr habe stets »die Bedingungen des Wachstums der Diktatur « kritisiert, was für Rasawi einer Gotteslästerung gleichkommt. Ajatollah Beheschti verkündete in einer Rede, daß das Programm der IRP davon ausgehe, die 
gesamte Bevölkerung und die Führung des Landes dem Islam näher zu bringen. Einen Erfolg der IRP sieht Rasawi a) in der starken Basis der Partei in der Bevölkerung und b) im konsequenten Umgang mit den Widersachern der Partei. Als Beispiel wird u.a. die Liquidierung der Volksmudjahedin erwähnt (Rasawi 1997: 202). Auch die Wahl landesweiter islamistischer Räte (»Kommunalwahlen«) wurde schon im Jahre 1980 von der IRP gefordert. Aber die Partei kam zu dem Schluß, daß in der revolutionären Phase wichtigere Probleme existierten als »Kommunalwahlen«. Diese wurden dann zwanzig Jahre später als Mobilisierungsstrategie aller Islamisten des Landes unter Khatami durchgeführt.

Schließlich löste Khomeini die Islamisch-Republikanische Partei am 3.6.1988 mit der Begründung auf, daß eine Partei in Kriegszeiten zu viel koste. Nach Kooroshy (1990: 120) sei die Partei tatsächlich von Fraktionskämpfen geplagt gewesen, so »daß ihre Weiterexistenz zu weiteren politischen Polarisierungen innerhalb der politischen Führung hätte beitragen können «. Kooroshy teilt die Konfliktparteien innerhalb der IRP in zwei Hauptgruppen ein: Eine Gruppe, die für die Verstärkung der Rolle des Staates in der Wirtschaft und eine verstärkte Kontrolle über den Außenhandel eintritt und eine andere Gruppe, die ein unbegrenztes Eigentumsrecht befürwortet und sich auf die ursprünglichen islamischen Lehren beruft (Kooroshy 1984:17). Die IRP hat dank der staatlichen und der parastaatlichen Organe erreicht, alle andersdenkenden Persönlichkeiten, Gruppen, Organisationen, Parteien und Minderheiten aus dem Land zu verjagen, in den Gefängnissen zu eliminieren oder zum Schweigen zu bringen.

\section{Wirtschaftsliberalisierung und Stärkung der Mullah-Diktatur}

Khomeini starb am 3. Juni 1989, ein Jahr nach dem Ende des achtjährigen Krieges gegen den Irak. Im November 1985 war Ajatollah Montazeri vom Expertenrat als Nachfolger Khomeinis gewählt worden (Schirazi 1997:73). Khomeini disqualifizierte ihn jedoch, weil er politisch unfähig sei. Erwartungsgemäß entstand infolge des Machtvakuums ein Führungskonflikt. Zwei Ajatollahs, Ali Khamenei und Akbar Haschemi Rafsandjani, bildeten eine neue Doppelspitze im iranischen Gottesstaat. Khamenei übernahm einen Tag nach Khomeinis Tod das Amt des Stellvertreter Gottes im Iran und am 28. Juli wurde Rafsandjani Parlamentspräsident. Diese Doppelspitze widerspiegelt einen alten Konflikt innerhalb der Kaste der herrschenden Geistlichkeit, der am deutlichsten in der IRP zum Ausdruck gekommen war und nun zum Hauptkonflikt der herrschenden Mullah-Riege werden sollte. In der Rolle des Hardliners ist Khamenei stets bedacht, eine wirtschaftliche Öffnung nur solange zu dulden, wie die Alleinherrschaft der Geistlichkeit nicht gefährdet wird. Rafsandjani wie sein Nachfolger Khatami überneh- 
men die Rolle der Wirtschafts-»Liberalen«, die das Ziel einer wirtschaftlichen Stärkung des Gottesstaates verfolgen. Und nur gemeinsam können die zwei Ajatollah-Fraktionen, die Hüter des Gottesstaates und die um die Weiterexistenz sich sorgenden »Wirtschaftsliberalen «, die Dynamik der Macht potenzieren und als ein islamistisches Doppelgespann die Diktatur stärken und aus der Isolation herausführen.

Die Politik der »Liberalisierung « der Wirtschaft war nicht gänzlich neu. Erinnert sei an den argentinisch-iranischen Vertrag bei der Umrüstung eines Kernreaktors der Teheraner Universität im Mai 1988, bereits zu Lebzeiten Khomeinis, und an den Besuch des sowjetischen Außenministers Schewardnadze bei Khomeini im Februar 1989. Iran war ein guter Handelspartner in Sachen Waffengeschäfte und wurde mit MIG-29 und SU-24 aufgerüstet (Hunter 1993: 186). Diese Beispiele sollen zeigen, daß die Politik der »Liberalisierung « nur in Form der Intensität neu war, denn Khomeini hatte stets verlautbaren lassen, daß im Interesse des Gottesstaates auch mit dem $»$ Teufel« verhandelt werden dürfe.

Nach einem verlustreichen Krieg war das Land international isoliert und geschwächt. Das islamische Regime entschied sich für wirtschaftliche Reformen, um die Weiterexistenz des Welayat zu sichern. Khamenei setzte sich für die ideologische Legitimation der Islamischen Republik ein und Rafsandjani damals, wie Khatami heute stehen für die begrenzte Modernisierung der Wirtschaft. Ein kurzer Überblick soll die Abhängigkeit der wirtschaftlichen Entwicklung des Gottesstaates vom internationalen Kapital verdeutlichen:

Nach dem Ende der tiefen Rezession von 1980 lebte die Wirtschaft in den Kriegsjahren 1981 bis 1986 wieder auf, dann folgte zwischen 1987-89 eine erneute Rezession (Amuzegar 1992: 417). Nachdem jegliche Opposition in mehreren Hinrichtungswellen 1979/82 und 1988 liquidiert worden war, wurde der ökonomische Niedergang zur größten Gefahr für die Machthaber. Trotz der vielen Versprechungen Khomeinis hatte sich die soziale und wirtschaftliche Lage Ende der achtziger Jahre für die Mehrheit der Bevölkerung dramatisch verschlechtert. Die iranische Zentralbank mußte im Frühjahr 1991 die Import- und Devisenbestimmungen lockern, um die Verarmung und den wirtschaftlichen Niedergang einzudämmen (Kooroshy 1997: 71). Um die Jahreswende 1991/92 belief sich die Auslandsverschuldung des Iran auf 16 Mrd. US \$, hinzu kamen Lieferantenkredite in Höhe von 4-5 Mrd. US \$ (Hermann, März 1992: 4). Trotz Auslandshilfe der wichtigsten westlichen Staaten konnte die Lohnentwicklung Anfang der 90er Jahre nicht mit der Teuerungsrate Schritt halten. Ende 1992 kletterte die Auslandsverschuldung auf 25 Mrd. US \$ (Hermann, Dezember 1992: 4f). 1993/94 gab es eine Geldentwertung um ca. 50\%. Die konjunkturelle Entwicklung der folgenden Jahre litt unter der hohen Inflation in Verbindung mit einem abnehmenden Außenwert des Rial (Espey, März 1994: 6). 
Der kurze Frühling von 1994, der mit dem Namen Rafsandjani in Verbindung gebracht wurde, dauerte jedoch nur wenige Monate (Hermann 1994: 541). Mitte der 90er Jahre wurde die wirtschaftliche Liberalisierung des Landes vom Religionsführer Khamenei gestoppt, der auf die Beachtung sozialer Aspekte bei wirtschaftlichen Unternehmen hinwies. Khamenei warnte vor einer staatlichen Wirtschaftspolitik, die sich bloß an hohen Wachstumsraten orientiert. Er forderte mehr wirtschaftliche Autarkie und größere eigene Anstrengungen in Richtiung einer islamischen Planwirtschaft. Auch der Regierung von Rafsandjani war bewußt, daß staatliche Eingriffe unvermeidbar sind. Immerhin betrug die Auslandsverschuldung 1993/94 insgesamt ca. 33 Mrd. US \$ (Espey, März 1995: 12). Drei Freihandelszonen sollten Investitionen internationaler Konzerne ermöglichen. Dort konnte der ausländische Investitionsanteil bis zu 100\% erreichen und für 15 Jahre wurde eine Befreiung von Einkommens- und Kapitalertragssteuern gewährt. Bis Ende 1995 sanken zwar die Auslandsschulden um 5 Mrd. US \$ auf 28 Mrd. US \$, aber es gab keine konjunkturelle Belebung.

Präsident Clinton verhängte am 8.5.1995 ein umfassendes Wirtschaftsembargo. Schon im März 1994 hatte Clinton die Kooperation der US-Firmen Conoco mit der National Iranian Oil Company (NIOC) untersagt. USamerikanische Firmen lieferten noch über Tochtergesellschaften in Europa und Asien in den Iran, US-Gesellschaften zogen ihr Personal aber aus dem Land ab. Da die Ankurbelung der Wirtschaft durch das Embargo im Jahr 1995 erschwert wurde, benötigte der Iran neue Kredite (Espey, Juli 1995: 4). Auch 1996 bestanden nur geringe Aussichten für eine Verbesserung der ökonomischen Lage. Die Privatisierung des staatlichen Industriesektors blieb trotz aller Bemühungen der wirtschaftsliberalen Mullahs aus, zumal ein Großteil der formal privatisierten Betriebe weiterhin den staatlichen Banken, öffentlichen Investitionen und religiösen Stiftungen gehörten. Deutschland und Japan gehörten 1996 zu den größten Gläubigern der zunächst auf 15 Mrd. US \$ reduzierten Auslandsverschuldung. Teilweise wirkten sich die US-Sanktionen bereits aus. So stoppte Japan z.B. die Auszahlung der zweiten Kreditrate für den Bau des Wasserkraftwerkes Karun4 in Höhe von 430 Mio. US \$ (Espey März 1996: 8). Die deutschen Direktinvestitionen wuchsen im Jahr 1996 auf 52 Mio. DM, was eine Steigerung von 11 Mio. im Vergleich zum Vorjahr bedeutete (Bundesbank Mai 1998: 25). Die iranische Regierung beschleunigte die industrielle Entwicklung und erhöhte die Zahl der Joint Ventures im Jahre 1995/96 auf 14 neue Vorhaben in Höhe von 352 Mio. US \$. Die Verschuldung war im Laufe des Jahres 1996 wieder auf 22 Mrd. US \$ gestiegen. Der Sprecher des Majlis Nateg Nouri und Khamenei kritisierten die hohe Verschuldung des Landes, weil lediglich eine kleine Schicht von »Profiteuren« begünstigt worden sei (Espey 1996: 10). Dank der hohen Exporterlöse (vor allem durch Öl) von 
knapp 19 Mrd. US \$ befand sich die Wirtschaft des Gottesstaates im Jahr 1997 allerdings wieder im Aufwind.

Im Jahr 1997 traten die US-Sanktionen in Kraft. Betroffen waren Firmen, die innerhalb eines Zeitraums von zwölf Monaten mehr als 40 Mio. US \$ in Irans Öl- und Gassektor investierten. Dennoch liefen die Wirtschaftsbeziehungen der westlichen Industrienationen ungestört weiter. Zu den Gewinnern des Geschäfts mit der Mullah-Diktatur gehörten u.a. die EU-Länder Frankreich, Italien und Großbritannien. Korea meldete einen enormen Anstieg des Iran-Geschäftes. Die deutschen Lieferungen fielen dagegen im Vergleich zu 1995 um 9,5\% (Espey 1996: 22). 1997 wurden die auf 10 Mrd. US \$ angestiegenen Devisenreserven langsam abgebaut, damit die sinkenden Öleinnahmen kompensiert und die Importe gesteigert werden konnten. Alle Haupthandelspartner des Iran profitierten von den steigenden Einfuhren des Gottesstaates. Die Auslandsverschuldung war 1997 rückläufig und betrug 18,8 Mrd. US \$. Die Mullahs haben Glück. Die USWirtschaftssanktionen verfolgen nicht ernsthaft das Ziel, die Europäer zu stoppen. Die USA wollten lediglich stärker in die Iran-Politik der EU involviert werden. Jenseits der wirtschaftlichen Beziehungen beschlossen die EU-Länder am 29.4.1997 eine Einschränkung der politischen Zusammenarbeit. Die gesamten EU-Lieferungen nach Iran stiegen 1996/97 um 8\% auf 4.8 Mrd. US \$. (Espey 1997: 18)

In den Jahren 1997/98 hatte sich die Zusammenarbeit mit dem Westen endlich gelohnt. Das Bruttoinlandsprodukt (BIP) der Mullah-Diktatur stieg um ca. 5\%. Der neue Präsident Khatami hat zwar noch kein Wirtschaftsprogramm, er will aber keine so starken Liberalisierungsmaßnahmen, die soziale Kosten verursachen würden, obwohl er die Relevanz des privaten Sektors hervorhebt. Khatami will auch die Rahmenbedingungen für Unternehmer verbessern, um mehr Arbeitsplätze zu schaffen. Im Jahr 1997 haben die Inlandsinvestitionen um 7,4\% zugenommen, die Auslandsinvestitionen wuchsen ebenfalls (Espey 1997: 27). Die Aufwärtsbewegung der iranischen Ökonomie konnte sich im Jahr 1998 aber nur langsam fortsetzen, da die Ölpreise stark gesunken sind, was die Ölexporterlöse 1998 um 4 Mrd. US \$ verringerte. Die Auslandsverschuldung belief sich auf 14,3 Mrd. US \$, die Devisenreserven wurden auf 6 Mrd. US \$ abgebaut. Die Inflationsrate bewegt sich zwischen 17-25\%. Auch unter Khatami wurden die staatlichen Betriebe nicht privatisiert. Ähnlich wie Rafsandjani, der in den Jahren 1994/95 und 1995/96 mit einer starken Inflation zu tun hatte, will Khatami gegen »Spekulanten « hart vorgehen (Espey 1998:21). Auch die Arbeitslosigkeit wächst stark, jährlich müßten 800.000 neue Arbeitsplätze geschaffen werden, was sich unmöglich realisieren läßt.

Italien und Japan zählten im Jahr 1998 zu den Hauptgewinnern der liberalen Handelsbeziehungen mit dem Mullah-Regime. Italien exportierte in 
Höhe von 4,5 Mio. US \$, d.h. 6\% mehr als im Vorjahr (Espey, Dezember 1998: 38). Seit März 1999 dürfen ausgewählte ausländische Unternehmen im Land Investitionen mit einem mehr als 50\%igen Eigenanteil vornehmen (Wahdathagh 1999d: 19). Aus Deutschland sollen die Mullahs sechs neue Hermes-Kredite, mit einem Volumen von über 200 Mio. DM bekommen (Ludwig/Mascolo 1999: 76).

Auch die Mullah-Diktatur ist trotz all der Forderungen nach Autarkie vom Westen abhängig. Die Abhängigkeit wächst mit dem Zwang zur wirtschaftlichen Entwicklung. Denn die Zeitbomben der nächsten sozialen Aufstände, wie die Hungerrevolten in Islamabad, die 1995 blutig niedergeschlagen wurden, ticken weiter. Die wirtschaftliche Entwicklung führt zu einer verstärkten politischen Kontrolle, denn die westliche Technologie bringt auch die westliche Kultur mit und genau dies versuchen beide Fraktionen des Mullah-Regimes zu verhindern, wie die Satelliten-Diskussion gezeigt hat. Die Iraner sollen keine westlichen Fernsehsender empfangen, BBC und Radio-Israel hören Hunderttausende unter den Kopfkissen.

\section{Der iranische »Gottesstaat « als totalitäre Diktatur}

Im Anschluß an Hannah Arendt lassen sich vier zentrale Kriterien für eine totalitäre Diktatur angeben, die auch auf die gegenwärtigen politischen und sozialen Konfigurationen der »Islamischen Republik Iran« zutreffen.

\section{Masse und Bewegung}

»Totalitäre Bewegungen sind Massenbewegungen« (Arendt 1986: 499). Totalitäre Regierungen werden von der Unterstützung der »Massen « getragen. Die Masse ist eher fasziniert von der fanatischen Ideologie als manipuliert. Das ganze Volk wird politisiert. Die Aufteilung in »uns« und die »anderen « zwingt die Masse zur Loyalität. Indem der Khomeinismus eine kanonische Geltung bekam und die Rückkehr zum Kalifat als das Heilmittel schlechthin betrachtet wird, wird die Welt in Gläubige und Ungläubige geteilt und die Religiosität des einfachen Menschen funktionalisiert.

Nach Arendt ist das praktische Ziel der Bewegung möglichst viele Menschen in der Bewegung zu organisieren, wobei die Bewegung aber mehr ist als eine Parteimitgliedschaft: sie vertritt die Person in ihrer personalen Totalität und befriedigt deren spirituelle Bedürfnisse (Arendt 1986: 540).

Neben kultischen Zeremonien erfolgt die Mobilisierung der islamistischen Bewegung auch über die landesweiten Wahlen. Khatami, der »ErneuererTraditionalist « (Wahdathagh 1998d) hatte im Zusammenhang der 20-Jahr Feier der islamischen Revolution die Wahlen zu den islamistischen Räten forciert, weil er davon ausgeht, daß die Herrschaft der Geistlichkeit eine Massenbasis braucht. Die Wahlen zu diesen Räten sind keine Kommunal- 
wahlen, denn sie dienen lediglich zur Mobilisierung der islamistischen Masse.

Die Bildung von Räten ist keine Erfindung des neuen iranischen Präsidenten Khatami und erst recht kein Konzept zur Demokratisierung; sie war bereits eines der Ziele der IRP. Auch Khomeini war schon ein Verfechter der islamischen Räte. Die khataminahe Zeitung des ehemaligen Bürgermeisters von Teheran schrieb, daß die Wahlen die absolute Macht im Staat dezentralisieren würden. Khomeini habe die Überwindung der diktatorischen Macht des Schahs mit der Schaffung eines auf den richtigen Weg führenden islamischen Rätesystems gewollt (Hamschahri, 6.1.99).Auch Hamschahri gesteht ein, daß die Wahl der Räte nicht in Zeiten der Konsolidierung der Revolution und des Krieges hätte durchgeführt werden können. Diese Form der Dezentralisierung der Macht dient tatsächlich ihrer Konsolidierung auf der Grundlage einer gestärkten islamistischen Bewegung. Die Masse wird zum Träger der absoluten Macht im Staat. Noch im Januar versuchte der etatistische Flügel, der als »rechts«-islamistisch bezeichnet wird, die Rechte der Räte einzuschränken. Dieser Flügel vertraut noch nicht der ideologischen Stärke der Basisbewegung. Im Majlis forderten sie die stärkere Anbindung der islamischen Räte an den Staat.

In 65 Städten, 8 Kleinstädten und 2081 kleinen Landbezirken wurden Ende Februar 1999 insgesamt 2154 islamistische Räte gewählt. Im Zuge der Wahlen wurde Ajatollah Hodjateseslam Kadivar verhaftet, weil er die Disqualifizierung Hunderter islamistischer Kandidaten kritisiert hatte; diese waren nicht völlig auf der »Linie des Imam Khomeini«. Ajatollah Kaschani, vorläufiger Freitagsimam von Teheran verkündete, daß der religiöse Glaube der Bevölkerung nicht ausgelöscht sei (Hamschahri, 5.1.99). Die Wahlen wurden als ein Sieg der khomeinistischen Revolution gefeiert. Tatsächlich haben sich aber weniger Menschen beteiligt, als erwartet wurde. Es wurden nur 15.343.237 Stimmen abgegeben, obwohl das Wahlalter auf 15 Jahre herabgesetzt worden ist - bei einer Einwohnerzahl von mehr als 60 Millionen Menschen (IRNA, 1.3.99).

Von Islamisten wird der Aufbau eines islamischen Rätesystems als ein weiterer Schritt in Richtung »islamischer Demokratie « angesehen (Hamschahri, 25.1.99). Wenn die Macht zu einem bestimmten Maß $\gg$ Personen gehören soll, die mehr Wert als andere haben, die mehr motiviert sind, und stärkere Zielorientierung als andere haben« (ebd.), paßt dies in das Konzept von Khatami, der von der Stärkung der islamistischen Kräfte, anstelle von der als unmöglich angesehenen vollkommenen Liquidierung der Gegner spricht. Khatami schrieb:

»Der Weg der Bewahrung und des Schutzes des 'Körpers' und die Gewährung einer Hilfe, die ihn dazu bringt unabhängig und selbständig auf eigenen Beinen zu stehen, ist nicht der Versuch, jede Mikrobe und jeden Virus daran hindern zu wollen, sich dem Körper anzunähern, 
sondern wir müssen etwas tun, damit der lebendige Körper und der Organismus gegen die Mikrobe Widerstand leisten. In Bezug auf die Gesellschaft und die menschliche Ordnung ist es nichts anders. Eine lebendige Gesellschaft muß die Gedanken der anderen und der Andersdenkenden kennen; das Kennen allein reicht auch nicht aus, diese Gesellschaft muß mit einer Logik und einem Denken ausgerüstet sein, das stärker und fruchtbarer ist als das der anderen« (Khatami 1997: 153).

Khatami bezeichnete die Wahlen stets als eine »ethische, islamische und menschliche Pflicht« (Hamschahri, 25.1.99). Rechte, Linke, Moderate müßten zusammenwachsen und die Kommunalwahlen könnten die nationale Entschlossenheit (Asm) der Bevölkerung beweisen, wenn die kontrollierenden Instanzen im Rahmen der Wahlen agieren würden. Die Wahlen würden die Akzeptanz und die Legitimität der Regierung noch mehr stärken. Er fügte aber auch hinzu, daß das Problem der Räte schon im Koran erwähnt worden sei, was bisher leider keine Berücksichtigung gefunden habe. Für Khatami sind die Wahlen ein Hauptziel und die Fortführung der islamischen Revolution im nationalen Rahmen (Hamschahri, 29.1.99). Keine Regierung werde auf Dauer Bestand haben können, wenn die Bevölkerung diese nicht mittrage. Die »Madani-Gesellschaft « könne nur existieren, wenn unterschiedliche »Kanäle « existieren könnten. »Madani-Gesellschaft « wird fälschlicherweise als Zivilgesellschaft übersetzt, die Mullahs meinen damit lediglich die Zivilität der islamistischen Untertanen in Welayate Fagih, der Herrschaft der Rechtsgelehrten (Wahdathagh 1998d). Die Meinungsvielfalt überschreitet damit nicht die der IRP. Dementsprechend entstammen die legalen politischen Kräfte auch dem Dunstkreis des Islamischen Revolutionsrates und der IRP.

\section{Ideologie und Propaganda}

Nach Arendt setzen totalitäre Diktaturen ihre ideologischen »Doktrinen und die aus ihnen folgenden praktischen Lügen mit Gewalt in die Wirklichkeit « um (Arendt 1986: 546). Die Propaganda ist die Voraussetzung zur Gewinnung von Massen, Mob und Elite. Nachdem die Opposition/Gegenpropaganda in kurzer Zeit ausgelöscht worden ist, gilt es nur noch die eigene Ideologie mit Terror zu verwirklichen. Die »Überlegenheit « der eigenen Doktrin begründet, die Unterdrückung der »minderwertigen « Lehren. Während im Bolschewismus der Führer als Vollstrecker angeblich historischer und im Nationalsozialismus als Vollstrecker angeblich biologischer Gesetzmäßigkeiten auftritt, beansprucht der Khomeinismus Vollstrecker göttlicher Ziele zu sein und stets wird von den Führern das Verschwinden der gegnerischen Gruppen prophezeit. Im religiösen Totalitarismus werden die Feinde Gottes zum Opfer, bei dessen Exekution die ideologische »Prophezeiung « sich bewahrheitet: »Ist die Exekution der Opfer dann eingetreten, so wird die 'Prophezeiung' zu einem nachträglichen Alibi: Es ist nur einge- 
treten, was vorausgesagt war (Arendt 1986: 557). Im Iran wird die Rolle der Opfer von den Kommunisten, den säkularen Gruppen und der religiösen Gemeinschaft der Bahá'í eingenommen.

»Wir glauben an Welayat « schrieb Khomeini (1997: 14). Diese khomeinistische Idee verfolgt den Eslame nabe Mohammadi, den puren mohammadanischen Islam als Herrschaftsideologie. Als Modell gilt das Kalifat von Imam Ali, der von Sunniten und Schiiten gepriesen wird. Für Khomeinisten gibt es »teuflische Mächte« und die »Anhänger Gottes«, wobei der islamische Führer auch mit dem Teufel verhandeln darf, wenn es dem eigenen Vorteil dient. Nach Khomeini ist Brüderlichkeit und Einheit der Muslime »nur in einem Staat der islamischen Welt, in der islamischen Republik Iran, verwirklicht« (Buchta 1997: 65). Diese Einheit wird mit der Identifizierung des politischen Feindes bei kultischen Anlässen wie dem Jerusalem Tag gefeiert (Wahdathagh, Elsässer 1998a). Die khomeinistische Lehre hat eine Möglichkeit für die Annäherung zwischen Sunniten und Schiiten geschaffen, dank Khomeini dürfen »nun auch die schiitischen Geistlichen ihren Staat haben...« (Tibi 1996: 115).

Der Khomeinismus kann heute als eine politische Ideologie des Islamismus aufgefaßt werden. Khomeini führte eine radikale Änderung im Verständnis der sozialen Ordnung in der schiitischen Gesellschaft ein. Er unterstellte, daß der Mensch in einem Gottesstaat ein absolutes Maß an Gerechtigkeit erreichen könne. Die Grundlage dieser gerechten Gesellschaft könne nur auf der Basis der Gesetze des Koran erfolgen (Schmidtke 1987: 391). Wer sich nicht für das göttliche Gesetz einsetzt, ist ein Kafar, ein gottloser Frevler. So bleibt den Menschen nur ein Weg des Gehorsams gegenüber der irdischen Herrschaft. Die zentrale Idee der khomeinistischen iranischen Verfassung ist das Prinzip der Welayate Fagih, die Herrschaft des islamischen Rechtsgelehrten, von denen behauptet wird, daß sie eine Stufe absoluter Gerechtigkeit erreichen können. Ein solcher Absolutheitsanspruch wird auch vom religiösen Führer beansprucht und Khomeini konnte zu einer solchen führenden charismatischen Kraft werden, an die seine Anhängerschaft glaubte (vgl. Kimmel/Tavakol). Tatsächlich gab Khomeini den Menschen den Glauben, daß wer sich dem Islam seiner Prägung hingibt, ewig siegreich sein werde.

Dank eines gewaltigen Propagandaapparates des Ministeriums für Rechtsleitung (Wezarate Erschad), einer gleichgeschalteten Presse und der umfassenden Mobilisierung der Massen sowie der erfolgreichen Arbeit des Repressionsapparates, konsolidiert sich die Mullah-Herrschaft. Besonders in den Gefängnissen wirkt die Indoktrination, die dazu dient, einen neuen Menschen zu schaffen, den gläubigen Muslim (Baradaran 1998). Zu den Propagandazentren gehören neben örtlichen Moscheen und den theologischen Seminaren der Hoseje Elmiye in Ghom das Freitagsgebet an der Te- 
heraner Universität. Der religiöse Führer ernennt die Freitagsimame persönlich. Auch sind die größten Konzerne in der Hand islamischer Stiftungen.

Die Glaubwürdigkeit der Islamischen Republik Iran nimmt sowohl bei der eigenen Bevölkerung als auch international immer mehr ab, obwohl der Traditionalist Khatami immer wieder versucht, die Bevölkerung propagandistisch zu mobilisieren, wie zuletzt bei der Kommunalwahl. Die Angst des religiösen Führers und des Präsidenten, daß bei zu starker Öffnung die Bevölkerung nochmals auf die Straße gehen könnte, ist berechtigt. Der Herrschaftsapparat würde dann langsam erodieren und ein ähnliches Schicksal wie die ehemals kommunistischen Staaten erleiden (Halliday 1994).

Die iranischen Intellektuellen und Politiker, die auf Khomeini setzten, haben keine Lehren aus der neueren Geschichte Irans gezogen. So ließ der Enthusiasmus im »Frühling der Freiheit« den Intellektuellen und ihren politischen Organisationen keine Zeit, um sich an die historischen Massenmorde der schiitischen Geistlichkeit der letzten 150 Jahre zu erinnern. Von den Pogromen gegen die Babi (Amanat 1989: 369f und Cole 1989: 28), von der Ermordung von rund 20.000 ihrer Anhänger unter aktiver Beteiligung des schiitischen Klerus wollte niemand etwas wissen. Bab gilt als der Vorläufer der postislamischen Bahá'í-Religion. Kaum jemand hatte das Buch Khomeinis gelesen, in dem er die Vernichtung der Bahá'í-Gemeinde angekündigt hatte (Khomeini 1997: 117). Niemand wollte an die reaktionäre Rolle der antiwestlich eingestellten schiitischen Geistlichkeit in der Zeit der konstitutionellen Bewegung von 1905-1906 erinnert werden (Momeni 1998b; Adjudani 1997), genausowenig wie an die Konflikte zwischen der Geistlichkeit und der »Nationalen Front« von Mossadegh, der 1953 durch einen von der CIA gesteuerte Putsch gestürzt wurde (Nirumand 1967).

Manche Intellektuellen fühlen sich von totalitären Bewegungen angezogen. Besonders, wenn »die alten Standards und Maßstäbe, ohnehin ihre Gültigkeit verloren haben « (Arendt 1986: 537). Im Rahmen dieses Beitrages kann nicht angemessen auf die Vielfalt der Intellektuellen, die sich mit graduellen Unterschieden von der religiösen Lehre oder dem antiimperialistischen Charakter der islamistischen Bewegung angezogen fühlen, eingegangen werden. Lediglich ein Beispiel sei genannt: Sorusch und Khatami gehören zu den Exponenten der Revolution. Sorusch, ein Staatstheoretiker der Islamischen Republik Iran (Enfag 1996) stellt wie Khatami die Unfehlbarkeit der Worte des Führers in Frage, eine solche Unfehlbarkeit gebe es nur Gott. Nicht in Frage gestellt wird aber der absolute Herrschaftsanspruch der Geistlichkeit. Dennoch wurde Sorusch in der deutschen Presse zuweilen mit Luther verglichen und Khatami gilt noch heute als Reformer. 


\section{Geheimpolizei und paramilitärische Einheiten}

Im Mykonos-Urteil wurde die Funktion der iranischen Geheimpolizei, VEVAK folgendermaßen beschrieben:

»Erste Maßnahmen, die die spätere Liquidierungsentscheidung vorbereiteten, leitete Ali Fallahian als Minister des VEVAK ein. Dem VEVAK oblag generell die nachrichtendienstliche Sammlung von Informationen über oppositionelle Gruppen, Parteien und Personen im In- und Ausland. Fallahian beauftragte Mohammad Hadi Hadavi Moghadam mit der Beschaffung von aktuellen Informationen über die DPK-I, insbesondere über ihre führenden Personen, und mit der Erarbeitung von Lösungsvorschlägen « (Archiv für Forschung und Dokumentation IranBerlin, 1998: 22).

Neben der Liquidierung von Oppositionellen im Ausland liegt die Hauptfunktion der Geheimpolizei und paramilitärischer Einheiten in der Unterdrückung der eigenen Bevölkerung. Dem Polizeiapparat fällt die Aufgabe $\mathrm{zu} »$ die Transformation der Tatsächlichkeit in die Fiktion zu überwachen« (Arendt 1986: 647).

Ein Instrument waren die nachrevolutionären Schauprozesse, die nach Abrahamian (1996: 81) mit denen unter Stalin und der »Gehirnwäschekampagne « der chinesischen Kulturrevolution zu vergleichen sind. In ihnen sollten Schuldbekenntnisse die Verwandlung der gesamten Bevölkerung in eine einheitliche Bewegung bezeugen (Baradaran 1998). Im Januar 1999 gab es eine offene Diskussion über den iranischen Geheimdienst. Khatami konnte nicht mehr verheimlichen, daß sein VEVAK für die Morde an dem populären Politiker Foruhar beteiligt war. Khatami »säuberte « sein Innenministerium und stellte sich schließlich mit Khamenei hinter den berüchtigten Apparat (Wahdathagh 1999a: 20).

Die Geistlichkeit selbst und die revolutionären Islamisten bildeten eine Armee von Geheimdienstlern. Sie wurden in den gesamten Staatsapparat geschleust, um in allen Bereichen die Islamisierung zu lenken und Abweichungen verhindern.

Die Pasdaran zählten zu den gefürchtesten islamistischen Militärapparaten. Rußland soll direkt an die insgesamt 900.000 Mann zählenden Einheiten Waffen geliefert haben. Die Pasdaran stellen bis heute, trotz der Integration in die Armee, eine Armee in der Armee dar. Die Armee wurde vernachlässigt und zugunsten der neuen ideologischen Polizei degradiert. Die zu Zeiten des Schahs westlich ausgebildete Armee ist allerdings längst gegenüber der klerikalen Regierung loyal (Cann/Danopoulos 1997: 274). Auch die jugendlichen Bassij-Einheiten, eine Armee von Freiwilligen, stellt einen starken Machtfaktor dar. Die islamischen Revolutionskomitees wurden inzwischen in die Polizei eingegliedert und erfüllen dort ihre Aufgabe der Islamisierung der Gesellschaft.

Die Geschichte der letzten zwanzig Jahre hat eindeutig gezeigt, daß die Khomeinisten keine Andersdenkenden dulden. »Objektive Gegner« werden 
aufgespürt und zu Verbrechern erklärt (Arendt 1986: 485). Der Terror wird systematisch durch Verfolgungs- und Mordwellen gestärkt. Tausende wurden in den letzten zwanzig Jahren in den iranischen Gefängnissen gefoltert, islamisiert, hingerichtet. Die Zwangsverschleierung der Frauen, der Hälfte der iranischen Bevölkerung, muß als eine geschlechtsspezifische Apartheidspolitik betrachtet werden (Moghissi 1998). Noch im Dezember letzten Jahres hat die Ermordung von mehreren legal arbeitenden, nicht oppositionellen Schriftstellern erneut bewiesen, daß die Islamische Republik unabhängige, öffentlich agierende Intellektuelle nicht toleriert (Wahdathagh 1998e). Die Angehörigen der Bahá'í-Religion sind weiterhin von einem Genozid bedroht. 1990 wurde die Existenz eines systematischen Plans zur Verfolgung der Bahá'í von der Menschenrechtskommission der UNO bestätigt, ein Plan, dessen Ziel die »Liquidierung aller kulturellen Wurzeln« im In- und Ausland ist (Iran-Report 1, 1995: 12). Im Iran dürfen die Bahá'í keine höheren Schulen besuchen. In den letzten Jahren hatte die Bahá'í-Gemeinde ein System privater Universitäten aufgebaut, die Fernstudien bei amerikanischen und kanadischen Universitäten organisierten, was unter Khatami zerschlagen wurde (Offener Brief 30.1.1999).

\section{Führerprinzip}

An der Spitze des Gottesstaates steht der Stellvertreter Gottes auf Erden, der charismatische religiöse Revolutionsführer. Nach Arendt sind die Massen ohne den Führer ein Haufen und »ohne die Massen ist der Führer ein Nichts« (Arendt 1986:527). Im Iran gibt es keine Souveränität des Volkes, denn die Umma ist das Volk Gottes und hat Gott und seinem Vertreter zu gehorchen. Dieser Vertreter ist ein Machtmonopolist. Nach der Verfassung ist er der Oberbefehlshaber der Streitkräfte (Art.110/4), entscheidet über den Einsatz der Streitkräfte (Art.110/5), wählt oder entläßt die Mitglieder des Wächterrates (Art.110/6a), ernennt den obersten Richter (Art.110 6b) und den Direktor des Medienrates des Landes (Art.110/6c); er ist befugt, den Generalstabschef (Art.110/6d), den Oberkommandierenden des Korps der islamischen Revolutionswächter (Art.110/6e) und den Oberkommandierenden der Armee und der Polizei (Art.110/6f) zu berufen und abzusetzen. Er steht über Judikative, Legislative und Exekutive (Art. 110/7), interveniert, wenn der Schlichtungsrat Konflikte nicht lösen kann (Art. 110/8), unterzeichnet die Ernennungsurkunde des Präsidenten nach seiner Wahl oder setzt ihn ab (Art. 110/9 und 10).

Nach der schiitischen Lehre darf eine irdische islamische Herrschaft erst beim Wiedererscheinen des verschwundenen 12. Imam errichtet werden. Die schiitische Geistlichkeit glaubt, daß der verborgene Imam noch nicht gekommen ist und wartet. Khomeini aber wollte nicht warten. Er stellte 
fest, daß Gott noch keine Person zur Errichtung der islamischen Herrschaft ausgewählt hat und fragte, sollen wir warten oder ist es nicht so, daß die Herrschaftsform, die in den Ursprüngen des Islam existierte, heute noch aktuell ist. Außerdem sei der Charakter des Gesetzes und der Gerechtigkeit in vielen Geistlichen vorhanden. Sie können zusammen kommen und eine gerechte Regierung bilden. Und wenn eine Person diese »edelsten « Eigenschaften inne habe, sei es die Pflicht aller Gläubigen ihr zu gehorchen (Khomeini 1997: 39f). Es gab aber auch innerreligiöse Legitimationskrisen. Denn der Führer muß eine Instanz der Nachahmung sein; da der Gläubige traditionell aber nur seine eigene »Instanz « nachahmen muß, stellt sich immer wieder die Frage, wen er nachahmen soll. Somit steht die »Zentralisierung der religiösen Macht (...) im Widerspruch zur althergebrachten Struktur des Klerus « (Chehabi 1993: 20). Dies ist auch ein Grund für die vielen Rivalitäten innerhalb der Kaste der Geistlichkeit. Khomeini war für die Islamisten eine integrative Autorität. Diese Funktion kann sein Nachfolger Khamenei nicht füllen. Um die Konflikte innerhalb der Geistlichkeit zu minimieren, sollte nach dem Tode Khomeinis der neue Führer seine Entscheidungen mit dem Schlichtungsrat, Majmae Taschkhise Maslehate Nezam nach Artikel 110 der Verfassung abstimmen (Meshkini 1989: 33). Das Führerprinzip wird somit im Konsens gestärkt; das Ziel bleibt die islamische Herrschaft trotz der verschiedenen Konzepte - mehr Marktwirtschaft oder mehr Staat. Daher dürfen die Differenzen innerhalb dieses Klüngels nicht als links oder rechts, »nicht als Aufbruch zum Pluralismus verstanden werden « (Wahdathagh 1999c).

Bereits oben wurde beschrieben wie die Islamisch-Republikanische Partei (IRP) zunächst die gesamte Macht im Land eroberte und im Sinne Arendts alle Staatsämter mit Parteigenossen besetzte (Arendt 1986: 647). Eineinhalb Jahre nach der Auflösung der Mullah-Partei versprach der ehemalige oberste Geheimdienstchef, Hojatoleslam Mohammad Reyschahri, im Oktober 1988, in Zukunft erneut politische Parteien zuzulassen. Ähnlich äußerte sich auch der damalige Innenminister und Mitbegründer der libanesischen Hezbollah, Ajatollah Ali Akbar Mohtaschemi. Auch nach der Wahl Khatamis gibt es wieder eine solche Propaganda. Doch noch immer sind die staatlichen Organe von den Khomeinisten besetzt und der »Pluralismus « innerhalb dieser Organe ist der »Pluralismus « der Meinungen der herrschenden Geistlichkeit und nicht der Pluralismus der Gesellschaft.

Bei den Präsidentschaftswahlen im Jahr 1997 brach der alte Konflikt, den Khomeini in der Islamisch-Republikanischen Partei so gefährlich fand, offen auf. Nateq-Nuri, der Rivale von Khatami, wurde von der militanten klerikalen Organisation Jameje-Rowhaniyate Mobarez (JRM) unterstützt (Fairbanks 1998). Zu den weiteren Unterstützern zählte Jamijate Motalefe Eslami, eine Organisation der Bazari. Khatami, wurde von der Ex-IRP- 
Fraktion der Majmae Rohaniune Mobarez (MRM), die im Gegensatz zu den reichen Bazaris für mehr soziale Gerechtigkeit auftreten und daher fälschlicherweise als Linksislamisten bezeichnet werden, unterstützt. Die MRM lehnen eine Öffnung vor allem zum Westen ab, und fordern eine strenge Islamisierung der Gesellschaft. Sie vertreten die mächtigsten Stiftungen des Landes und verteidigen die Ausweitung des Staatssektors. $\mathrm{Zu}$ den Gründungsmitgliedern dieser Organisation gehört auch der ehemalige Innenminister (1985-89), Ali Akbar Mohtaschempour, und Khoinia, Herausgeber der Zeitung Salam. Khatami wurde aber auch von den radikalen, nicht-klerikalen Organisation der Sazmane Modjahedine Eslami (Organisation der Kämpfer der Islamischen Revolution) unterstützt.

Diese verschiedenen Organisationen stellen unterschiedliche Fraktionen der khomeinistischen Ex-IRP dar. Keine der Gruppen tritt für demokratische Freiheitsrechte ein, die eben die Grenze zwischen rechts und links ausmacht. Dies zeigt, wie töricht die Einteilung der Islamisten in links, rechts und moderat ist, eher handelt es sich um Rivalitäten zwischen verschiedenen »Mullahstämmen «.

Der spektakuläre Sieg Khatamis beruht auch auf den Stimmen von 70\% der Revolutionsgardisten und der paramilitärischen Bassidj-Einheiten (Kooroshy 1998: 12). Tatsächlich beruht die Stärke von Khatami auf 20 Millionen Wählern. Viele von ihnen sehen seine pragmatischen West-Kontakte als einen möglichen Schritt zum Zerbröckeln des Regimes.

Vielfach wurde unterstellt, daß es mit der Wahl Khatamis zu »größerem Pluralismus und Demokratie« kommen werde (so Fairbanks 1997). Doch wird dabei übersehen, daß es sich nicht um den Pluralismus der Gesellschaft und erst recht nicht um die Demokratie-Forderungen der Bevölkerung handelt, sondern um eine Reflexion der Widersprüche innerhalb der herrschenden Minderheit.

Nach Steinbach war ein »entscheidender Schritt in Richtung auf die Islamisierung des politischen Systems (...) die Wahl einer Expertenversammlung « (Steinbach 1994: 256). In der Phase der Revolution hatten die Mitglieder des Expertenrates alle der IRP angehört. Sie legten in der Verfassung das Prinzip der »Herrschaft der Rechtsgelehrten« (Welayate Fagih) fest. Damit bekam die Kaste der Geistlichkeit eine zentrale Position in der Machtausübung. Die Geistlichkeit steht über dem Staat und gleichzeitig besetzt sie alle Funktionen des Staates. Zu den Aufgaben des 83köpfigen Expertenrates gehört nach Artikel 108 der Verfassung die Wahl des islamischen Führers (Buchta 1998: 61). Die Entscheidungen dieses Gremiums werden vom 12köpfigen Wächterrat überwacht (der alle Bereiche staatlicher Politik akzeptieren muß und gegen jedes Gesetz sein Veto einlegen kann). Bei der Wahl des Expertenrates wird der Kreis der Macht besonders deutlich: Der Expertenrat wählt den geistigen Führer, dieser bestimmt ei- 
nen Teil des Wächterrates, der wiederum die Kandidaten für den Expertenrat auswählt (Wahdathagh 1998c :22).

Neben dem Wächterrat gehört der Majlise Schoraje Eslami, die Versammlung des islamischen Rates, zur Legislative des Landes. Nach Art. 64 gehören ihm 270 Abgeordnete an, die nach einer Überprüfung des Wächterrates als Abgeordnete für 4 Jahre gewählt werden können. Beschlußfähig ist der Majlis, wenn 2/3 der Geistlichen anwesend sind. Rieck schreibt zwar, daß »dieses Parlament nie zu einem reinen Jasager-Verein degeneriert sei« (Rieck 1989: 549), verkennt aber, daß die Diskussionen eng begrenzt sind. Das Wort Parlament ist hier fehl am Platze, da die Abgeordneten keine Volksvertreter sind, sondern außer einer ganz kleinen Alibivertretung der religiösen Minderheiten, nur Vertreter der Kaste der Geistlichkeit umfaßt.

\section{Fazit}

Die politische Religion des Khomeinismus stellt ein komplexes Glaubenssystem dar, das aber auch mit traditionellen schiitischen Konzeptionen in Widerspruch steht. Dieses Glaubenssystem liefert die ideologische Basis für eine »dritte « Spielart des Totalitarismus. Die wissenschaftlichen und technologischen Instrumente der »Islamischen Republik « machen im Spannungsverhältnis zwischen der Moderne und dem Kalifat als einer despotischen Herrschaftsform einen totalitären Staat erst möglich. Solange die Geistlichkeit als Stellvertreter Gottes auf Erden die staatliche Macht ausübt und die Unvernunft der khomeinistischen Ideologie heilig spricht, wird auch der Iran eine totalitäre Despotie bleiben. Mit Khatami hat sich diese Despotie lediglich ein neues Profil gegeben. Als Fazit kann daher mit Tibi festgestellt werden, daß es sich bei der Islamischen Republik Iran um eine rückwärtsgewandte Utopie vom »wahren «, weil »gerechten Islam«, in der »Substanz aber um eine neo-islamische Auflage moderner Diktatur « handelt. »In diesem Sinne ist die fundamentalistische Vorstellung von Hakimiyyat Allah/Gottesstaat sowie die neue Imam-Version eine Spielart des Totalitarismus« (Tibi 1996: 313).

\section{Literatur}

Abdolvand, Behruz; Borumand, Arasch; Pahlavan, Issa (1999): Barchi Molahezate Geopolitik dar rawabete Iran wa Amrika, in: Irane Farda, Nr.49

Abrahamian, Ervand (1996): Bekenntnis unter Folter: Gefängnisse und öffentliche Reaktionen im modernen Iran in: Internationale Liga für Menschenrechte (Hg.): Islamischer Fundamentalismus und Menschenrechtsverletzungen am Beispiel der Islamischen Republik Iran, Berlin

- (1993): History Used and Abused in: Khomeinism, Essays on the Islamic Republic, Berkeley

- (1998): Iran bejne do Enghelab as Maschrute ta enghlebe Eslami, Tehran

- Adjudani, Maschalah (1997): Maschruteje Irani wa Pisch Saminehaje Nazarijeje »Velayate Fagih" 
Amanat, Abas (1989): Resurrection and Renewal, The making of the Babi Movement in Iran, 1844-1850, London, Cornell University Press

Amuzegar, Jahangir (1992): The Iranian Economy Before And After The Revolution in: Middle East Journal, Volume 46, No.3, Summer

Archiv für Forschung und Dokumentation Iran-Berlin (Hrsg.) 1998: Mykonos-Urteil

Arendt, Hannah (1986): Elemente und Ursprünge totaler Herrschaft, München

Banisadr, Abolhassan (1979): Egetssade Towhidi, Tehran

Baradaran, Monireh (1998): Erwachen aus dem Alptraum, Union-Verlag, Zürich

Bernard, Cheryl/Khalilzad, Zalmay (1979): Secularization, Industrialization and Khomeinis islamic Republic in: Political Science quarterly, Number 2, Summer

Buchta, Wilfried (1998): Ein Haus mit vielen Herren: divergierende Machtzentren in der Islamischen Republik Iran in: Orient 39, 1998/1,

- (1994): Die inneriranische Diskussion über die islamische Einheit, in: Orient 35, 1994/4

- (1997): Die iranische Schia und die islamische Einheit 1979-1996, Hamburg

- (1995a): Die islamische Republik Iran und die religiös-politische Kontroverse um die marjaiyat, in: Orient 36

- (1995b): Mehdi Bazargan, in: Orient 36, 1995/4

Bundesbank (1998): Kapitalverflechtungen mit dem Ausland, Frankfurt Main

Cann, Rebecca/Danopoulos; Constantine (1997): The Military and Politics in a Theocratic State: Iran as Case Study, in: Armed Forces \& Society, Vol.24, No.2, Winter

Chehabi, Houchang E.(1993): Klerus und Staat in der Islamischen Republik Iran, in: Aus Politik und Zeitgeschichte, B33/93, Bonn 13.August

Chubin, Shahram (1982): Security in the Persian Gulf, IISS, Hampshire

Chubin, Schahram (1983): The Soviet Union and Iran, in: Foreign Affairs, New York Spring

Cole Juan R.I. (1989): Modernity and the Millennium, Columbia University Press New York

Dilger, Konrad (1982): »Die Gewalt des Rechtsgelehrten« im islamischen Recht, in: Zeitschrift für Vergleichende Rechtswissenschaft Heidelberg

Enfag, Wahid (1996): Schattenboxen mit der Geistlichkeit, in: Junge Welt 29.2.1996

Espey, Robert: (März 1994): Iran am Jahreswechsel 1993/94 in: bfai (Hg.), Köln

- (März 1995): Iran, Wirtschaftstrends zum Jahreswechsel 1994/95 in: bfai (Hg.), Köln

- (Juli 1995): Iran, Wirtschaftstrends zur Jahresmitte 1995 in: bfai (Hg.), Köln

- (März 1996): Iran, Wirtschaftstrends zum Jahreswechsel 1995/96 in: bfai (Hg.), Köln

- (1996a): Iran, Wirtschaftstrends zur Jahresmitte 1996, in: bfai (Hg.), Köln

- (1996b): Iran, Wirtschaftstrends zum Jahreswechsel 1996/97, in: bfai (Hg.), Köln

- (1997a): Wirtschaftstrends zur Jahresmitte 1997, in: bfai (Hg.), Köln 1997

- (1997b): Iran zum Jahreswechsel 1997/98, in: bfai (Hg.): Köln

- (1998): Iran zur Jahresmitte 1998, in: bfai (Hg.), Köln

- (Dezember 1998): Iran zum Jahreswechsel 1998/99 in: bfai (Hg.), Köln

Fairbanks, Stephan (1997): A New Era For Iran? In: Middle East Policy, Vol V, No.3

- (1998): Theocracy Versus Democracy: Iran Considers Political Parties, in: The Middle East Journal, Vol 52, No 1 Winter

Fukuyama, Frances (1992): Das Ende der Geschichte, München

Gellner, Ernst (1987): Warten auf den Imam, in: Schluchter, Wolfgang (Hrsg.): Max Webers Sicht des Islams, Ffm

Halliday, Fred (1994): An Elusive Normalization: Western Europe And The Iranian Revolution in: Middle East Journal, Volume 48, No 2, Spring

Hamschahri, Internetfassung der in Teheran erscheinenden persischsprachigen Zeitung

Hashim, Ahmed (1995): The Crisis of the Iranian State, Oxford 1995

Heinz, Wolfgang (1997): Interview geführt von Wahied Wahdathagh in: Jungle World 4.12.1997.

Hermann, Rainer, T. (März 1992): Iran am Jahreswechsel 1991/92 in bfai (Hg.): Köln

- (Dezember 1992) Iran am Jahreswechsel 1992/93 in: bfai (Hg.): Köln

- (1994): Von der Wirtschafts- zur Legitimationskrise, in: Orient 35, 1994/4

Hiro, Dilip (1987): Iran under the Ayatollahs, New York: Routledge \& Kegan Paul

Hobsbawm, Eric (1994): Das Zeitalter der Extreme, Wien

Hunter, Shireen T.(1993): Iran from the August 1988 Cease-Fire To The April 1992 Majlis Election 
Freedman, Robert: The Middle East after Iraq's Invasion of Kuwait, Florida

Huyser, Robert E.(1986): Putschen Sie, Herr General! - Wie Washington den Iran retten wollte, Reinbek

Internationale Liga für Menschenrechte, Iran-Gruppe (Hrsg.) 1995: Iran-Report 1, Berlin

Irna, Internetversion

Katouzian, Homa (1998): The Pahlavi Regime in Iran in: Linz, Juan J. Chehabi, H.E.: Sultanistic Regimes, London

Kennedy, Paul (1989): Aufstieg und Fall der grossen Mächte, Ffm

Khatami, Seyyed Mohammad (1997): Bime Modj, Tehran,

Khomeini, Ruhollah (1997): Velayate Fagih, Hokumate Eslami, Teheran

Kimmel, Michael S./Tavakol, Rahmat (1986): Against Satan: Charisma and Tradition in Iran, in: Swatos, William H.: Charisma, History and Social Structure, Connecticut/London

Kooroshy, Javad (1997): Die wirtschaftliche Dimension der deutsch-iranischen Sonderbeziehungen in: Blätter für deutsche und internationale Politik 1/97, Berlin

- (1998): Mohammad Khatami in: Orient 39, 1998/1,

- (1990): Wirtschaftsordnung der Islamischen Republik Iran, Hamburg

- (1984): Islamische Wirtschaftsordnung im Iran, in: Aus Politik und Zeitgeschichte, B42/84, Bonn

Leaman, George (1988): Die geheime Nebenregierung der USA, in: Das Argument 167

Lewis, Bernard (1988): Die islamische Revolution, in: Kursbuch 93, Berlin

Ludwig, Udo; Mascolo, Georg (19.4.1999): Kleinkrieg in der Nacht, in: Der Spiegel 16/1999, Hamburg

Luxemburg, Rosa (1985): Die Akkumulation des Kapitals, Berlin

Mahrad, Ahmad (1983): Iran nach dem Sturz des Schahs. Die provisorische Revolutionsregierung Bazargans, Frankfurt a.M.

Meshkini, Ali, Chairman: (1989) The Constitutional Review Council: The Constitution Of The Islamic Republic Of Iran, Tehran

Moghissi, Haideh (1998): Geschlechtstrennung als Apartheid, Interview geführt von Wahied Wahdathagh in: Jungle World, 25.3.1998

Momeni, Bagher (1998a): Engelabe Iran samineha wa hadafha, in: As modj ta Tufan, Essen

- (1998b): Din wa Dowlat dar Asre Maschrutiat, Schweden

Nasri, Farzeen (1983): Iranian studies and the iranian Revolution, in: World politics, New York July

Nirumand, Bahman/Daddjou, Keywan (1987): Mit Gott für die Macht, Berlin

Nirumand, Bahman (1967): Persien, Modell eines Entwicklungslandes oder die Diktatur der Freien Welt, Hamburg

Offener Brief gegen die Diskriminierung der Bahá‘í im Iran, (30.1.1999) in: Frankfurter Allgemeine Zeitung

Olianowsky (1979): Masael Moaser Asia wa Afria, Teheran

Parsipur, Schahrnusch (1996): Chaterate Zendan, (The Memoirs of Prisons) Schweden

Rasavi, Masud (1997): Haschemi wa Engelab, Tariche Siasye Iran as Engelab ta Djang, Tehran

Rieck, Andreas (1989): Das Parlament in der Islamischen Republik Iran, in: Orient 30, 1989/4

Rouleau, Eric (1980): Khomeinis Iran, in: Foreign Affairs, Fall

Schirazi, Asgahr (1991): Die neuere Entwicklung der Verfassung in der Islamischen Republik Iran, in: Verfassung und Recht in Übersee, 24.Jg. 2.Quartal 91, Hamburg

- (1997): The Constitution of Iran, Politics and the State in the Islamic Republic, London

Schmidtke, Sabine (1987): Modern Modifications in the Shii Doctrine of the Mahdi, in: Orient 28

Sciolioni, Elaine (1983): Iran's Durable Revolution, in: Foreign Affairs, Spring

Shirazi, Asghar (1992): Die Widersprüche in der Verfassung der Islamischen Republik vor dem Hintergrund der politischen Auseinandersetzung im nachrevolutionären Iran, Berlin

Sprung, Christopher (13.8.1998): Geheimprozesse und Hinrichtungen; Wie die Bahá'í im Iran verfolgt werden, Pressemittelung des Nationalen Geistigen Rates der Bahá'í in Deutschland, Ffm

Steinbach, Udo (25.5.1990): Der Islam im »Thermidor«, in: Aus Politik und Zeitgeschichte, B22/90 
- (1994): Iran, in: Steinbach, U. (Hg.), Der Islam in der Gegenwart, München

Tellenbach, Silvia (Hg.) (1996): Strafgesetze der Islamischen Republik Iran, Berlin

Tibi, Bassam (1996): Der wahre Imam. Der Islam vom Mohammad bis zur Gegenwart, München

Wahdathagh, Wahied; Elsässer, Jürgen (29.1.1998a): Fundis aller Länder vereinigt Euch! In: Jungle World, 29.1.1999

Wahdathagh, Wahied (1998b): Religiös legitimierte Tyrannei, in: die tageszeitung 2.2.1998

- (1998c): Mullahs wählen Experten wählen Mullahs, in: Jungle World, 28.10.1998

- (1998d): Mullahkratie und Wahnsinn, in: Vorgänge 144

- (1998e): Meine Täter, deine Täter: in: Jungle World, 23.12.1998

- (1999a): »Irregleitete Geheimdienstler«, in: Jungle World, 13.1.1999

- (1999b): Der Frühling der Freiheit ist ins Land gegangen, in: Frankfurter Rundschau 10.3.1999

- (1999c): Hochtechnisierter Gottesstaat, in: Ossietzky 13.3.1999

- (1999d): Irans Präsident Khatami auf Werbetour in Italien, Basar unter Strom, in: Jungle World, 17.3.1999

Wittfogel, Karl A.(1977): Die orientalische Despotie

Wright, Claudia (1980/81): Implications of the Iraq-Iran War, in: Foreign Affairs, Winter

Zonis, Marvin (1983): Iran: A Theory of Revolution From Accounts of the Revolution, in: World Politics, July 